\title{
Face Recognition Using Hierarchical Isomap
}

\author{
Dianting Liu, Zongying Ou, Guoqiang Wang, Shungang Hua, Tieming Su \\ Key Laboratory for Precision and Non-traditional Machining Technology of Ministry of \\ Education, Dalian University of Technology, P. R. China \\ diantingliu@yahoo.com.cn,ouzyg@dlut.edu.cn
}

\begin{abstract}
Nonlinear dimension reduction method Isomap has demonstrated promising performance in finding low dimensional manifolds from data points in the high dimensional input space. The Isomap method estimates geodesic distance between data points instead of taking the Euclidean distance and then uses multidimensional scaling (MDS) to induce a low dimensional embedding from the geodesic distance graph. However, since the original prototype Isomap does not discriminate data acquired from different classes, when concerned with multi-class data, several isolated sub-graphs will result in undesirable embedding. In this paper, a hierarchical Isomap algorithm is proposed for the multi-class data, which first computes within-class and between-class geodesic distances separately and the final embedding is obtained from the augmented geodesic distance matrix using MDS. The experimental results reveal a promising performance of the proposed algorithm.
\end{abstract}

\section{Introduction}

Classical dimensionality reduction methods can be classified into two main categories: linear and nonlinear methods. Two popular linear techniques are the methods of principal component analysis (PCA) [1] and MDS [2], which are efficient to find the true structure of the data when dealing with a linear manifold. In the PCA method, the optimal pdimensional subspace is selected by rotating the coordinate axes to coincide with the eigenvectors of the sample covariance matrix, keeping the $p$ axes along which the sample has the largest variance. The MDS method finds an embedding that best preserves the pairwise distances between data points. They are both simple to implement, efficiently computable, and guaranteed to discover the true structure of data lying on or near a linear subspace of the high-dimensional input space [3].
Besides the linear methods above mentioned, several nonlinear embedding methods have been designed to discover the structure of high-dimensional data and find their embedding in a low-dimensional Euclidean space. The LLE algorithm [4] uses a collection of local neighborhoods to guide the embedding. If the neighborhoods are small in quantity, they can be approximated as linear manifolds and the position of each point can be reconstructed as a weighted linear combination of its $\mathrm{k}$ nearest neighbors. The position of each point in the lower-dimensional space is determined by minimizing the reconstruction error therein. Solving an eigenvector problem, as in PCA, does this. The Isomap algorithm [5] uses geodesic distances between points instead of simply taking Euclidean distances, thus "encoding" the manifold structure of the input space into distances. The geodesic distances are computed by constructing a sparse graph in which each node is connected only to its closest neighbors. The geodesic distance between each pair of nodes is taken to be the length of the shortest path in the graph that connects them. These approximated geodesic distances are then used as input to classical MDS.

However, the above nonlinear methods are only suitable for the given sets of data from one single class. When we perform embedding directly using the Isomap algorithm to deal with multi-class data, unconnected sub-graphs will be formed, which will lead to dissatisfactory embedding. In order to cope with this problem, $\mathrm{Wu}$ [7] proposes to compute the between-class distance by using Euclidean distances of points belonging to different class. This method is available when handles the problem in which the number of the classes is relatively smaller than three [7]. When the number of classes becomes more, the classes may construct their own spatially intrinsic structure. Then Wu's method can't recover the classes' intrinsic structure of the high-dimensional data. Therefore, in this paper we propose a hierarchical Isomap, which uses a hierarchical geodesic distance 
instead of Euclidean distance to calculate betweenclass distances and within-class distances.

The rest of this paper is organized as follows. In Section 2, we introduce the hierarchical Isomap method that we proposed. In Section 3, we show the experimental results on hierarchical Isomap compared with original Isomap and extended Isomap. We conclude the paper in Section 4 .

\section{Proposed method}

Our method firstly calculates the central points for every class and constructs the geodesic distance matrix of central points. Then, we compute each within-class geodesic distance sub-matrix separately. Next, the geodesic distance between points belong to different classes is constructed by summing up their respective with-class geodesic distances and the geodesic distance between the two corresponding class central points. The final embedding is achieved by applying MDS on the augmented hierarchical geodesic distance matrix.

\subsection{Construct central point geodesic distance matrix}

In our work, we consider a set of $C$ class $\left\{Z_{1}, Z_{2}, \ldots\right.$, $\left.Z_{C}\right\}$; each class has $N$ points $\left\{x_{1}, x_{2}, \ldots, x_{N}\right\}$; point $m^{w}$ is defined as $w$-th class's central point and its value equates the mean value of all points in the $w$-th class, $w=1,2, \ldots, C$.

In the first step, we determine the neighbors of each central point $m^{w}$, based on Euclidean distances $d_{X}\left(m^{p}, m^{q}\right)$. Two methods are utilized to determine whether two central points are neighbors or not. One is based on $k$ nearest neighbor algorithm and the other includes all the points within some fixed radius $\varepsilon$ as neighbors. These neighborhood relationships are represented in a graph $G_{m}$

The second step is to estimate central point geodesic distance $d_{G-m}\left(m^{p}, m^{q}\right)$, where $m^{p}$ and $m^{q}$ are central points belong to different classes. Initialize $d_{G-m}\left(m^{p}, m^{q}\right)$ $=d_{X}\left(m^{p}, m^{q}\right)$, if $m^{p}$ and $m^{q}$ are neighbors, otherwise $d_{G-}$ ${ }_{m}\left(m^{p}, m^{q}\right)=\infty$. Then according to the Floyd-Warshall algorithm as Eq.(1), for each value of $o=1,2, \ldots, C$ in turn replace all entries $d_{G--m}\left(m^{p}, m^{q}\right)$ by $\min \left\{d_{G-m}\left(m^{p}, m^{q}\right), d_{G-}\right.$ $\left.{ }_{m}\left(m^{p}, m^{o}\right)+d_{G-m}\left(m^{o}, m^{q}\right)\right\}$.

$$
d_{G m}\left(m^{p}, m^{q}\right)=m i n\left\{d_{G m}\left(m^{p}, m^{q}\right), d_{G m}\left(m^{p}, m\right)+d_{G m}\left(m^{q}, m^{q}\right)\right\}
$$

We get central point geodesic distance matrix $D_{G-m}$ as follow:

$$
D_{G-m}=\left[\begin{array}{ccc}
d_{G-m}\left(m^{1}, m^{1}\right) & \cdots & d_{G-m}\left(m^{1}, m^{C}\right) \\
\vdots & \ddots & \vdots \\
d_{G-m}\left(m^{C}, m^{1}\right) & \cdots & d_{G-m}\left(m^{C}, m^{C}\right)
\end{array}\right]
$$

\subsection{Construct within-class geodesic distance sub-matrix}

The number of within-class geodesic distance subMatrix to be computed is $C$. We set the $w$-th class geodesic distance sub-matrix for example. First, determine the neighbors of each point $x_{i}$ (including $\mathrm{m}^{w}$ ) in the $w$-th class based on Euclidean distances $d_{X}\left(x_{i}, x_{j}\right)$ and $d_{X}\left(x_{i}, m^{w}\right)$. The second step is to calculate withinclass geodesic distance $d_{G}\left(x_{i}, x_{j}\right)$, where $x_{i}, x_{j}$ belong to the same class. If $i$ and $j$ are neighbors, initialize $d_{G}\left(x_{i}, x_{j}\right)=d_{X}\left(x_{i}, x_{j}\right)$, otherwise $d_{G}\left(x_{i}, x_{j}\right)=\infty$. Next, for each value of $k=1,2, \ldots, N$ in turn replace all entries $d_{G}\left(x_{i}, x_{j}\right)$ according to the Floyd-Warshall algorithm by $\min \left\{d_{G}\left(x_{i}, x_{j}\right), d_{G}\left(x_{i}, x_{k}\right)+d_{G}\left(x_{k}, x_{j}\right)\right\}$. We get the $w$-th class within-class matrix $D_{G}^{w}=\left\{d_{G}\left(x_{i}, x_{j}\right)\right\}$ :

$$
D_{G}^{w}=\left[\begin{array}{ccc}
d_{G}\left(x_{1}, x_{1}\right) & \cdots & d_{G}\left(x_{1}, x_{N}\right) \\
\vdots & \ddots & \vdots \\
d_{G}\left(x_{N}, x_{1}\right) & \cdots & d_{G}\left(x_{N}, x_{N}\right)
\end{array}\right]
$$

In the same way, the geodesic distance $d_{G}\left(x_{i}, m^{w}\right)$ between each point $x_{i}$ in the $w$-th class and its corresponding central point $m^{w}$ is computed according to the Floyd-Warshall algorithm by $\min \left\{d_{G}\left(x_{i}, m^{w}\right)\right.$, $\left.d_{G}\left(x_{i}, x_{k}\right)+d_{G}\left(x_{k}, m^{w}\right)\right\}$, where $k=1,2, \ldots, N . V^{w}=\left\{d_{G}\left(x_{i}\right.\right.$, $\left.\left.m^{w}\right)\right\}$ is central point geodesic distance vector:

$$
V^{w}=\left[\begin{array}{c}
d_{G}\left(x_{1}, m^{w}\right) \\
\vdots \\
d_{G}\left(x_{N}, m^{w}\right)
\end{array}\right]
$$

\subsection{Construct between-class geodesic distance sub-matrix}

We compute geodesic distance of between-class points $x_{i}$ and $x_{j}$, which separately belong to class $p$ and $q, p, q \in C$, shown as Figure 1. The geodesic distance $d_{G}\left(x_{i}, x_{j}\right)$ can be compute by

$$
d_{G}\left(x_{i}, x_{j}\right)=d_{G}\left(x_{i}, m^{p}\right)+d_{G-m}\left(m^{p}, m^{q}\right)+d_{G}\left(m^{q}, x_{j}\right)
$$


Then the between-class sub-matrix $D_{G}^{p-q}=\{$ $\left.d_{G}\left(x_{i}, x_{j}\right)\right\}$ can be constructed.

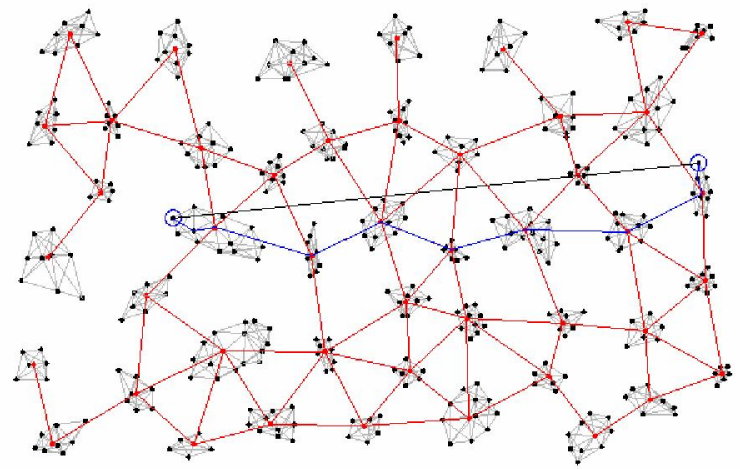

Figure 1. The two-dimensional embedding recovered by hierarchical Isomap toward multi-class data. Red lines represent the geodesic distances between class central points. Straight lines (black) represent simpler and clearer approximations to the true geodesic paths (blue).

\subsection{Construct hierarchical geodesic distance matrix}

After all within-class and between-class submatrices are computed respectively, the hierarchical geodesic distance matrix $D_{G}$ can be obtained by arraying sub-matrices as Eq.(6), where $D_{G}^{1}, D_{G}^{2}, \ldots, D_{G}^{C}$ are within-class sub-matrix, the other sub-matrices are between-class sub-matrices. Since $D_{G}$ is the symmetric matrix, $D_{12}=D_{21}$. The final embedding is achieved by applying MDS on the hierarchical geodesic distance matrix $D_{G}$.

$$
D_{G}=\left[\begin{array}{cccc}
D_{G}^{1} & D_{12} & \cdots & D_{1 C} \\
D_{21} & D_{G}^{2} & \cdots & D_{2 C} \\
\vdots & \vdots & \ddots & \vdots \\
D_{C 1} & D_{C 2} & \cdots & D_{G}^{C}
\end{array}\right]
$$

\section{Experimental results}

We test our proposed algorithm compared with original Isomap [5] and extended Isomap [7] on the open face database $\mathrm{JAFFE}^{1}$, which contains 213 face images corresponding to 10 Japanese females. Each person was recorded six basic facial expressions and a neutral face. 7 images from each person are selected for training, and the remaining 14 images are used for

\footnotetext{
${ }^{1}$ http://www.kasrl.org/jaffe_download.html
}

testing. All face images are geometrically normalized and aligned. Sample images from the set are shown in Figure 2.

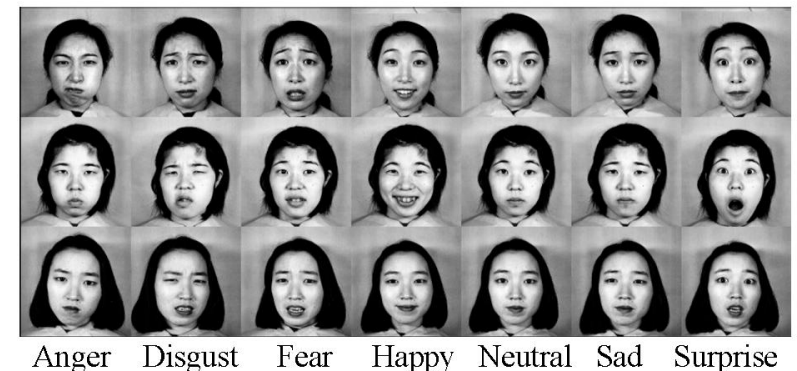

Figure 2. Samples from the Japanese females facial expression (JAFFE) image set

The experimental results are shown in Figure 3. In the experiment, Support Vector Regression (SVR) [8] is used to define a mapping from the input to the output space for the training data. Then for the test data points in the high-dimension space, the location of the corresponding points in lower-dimension space is computed by SVR. Finally, Support Vector Machine (SVM) [9] is employed for final classification and the polynomials kernel is selected for SVM decision functions.

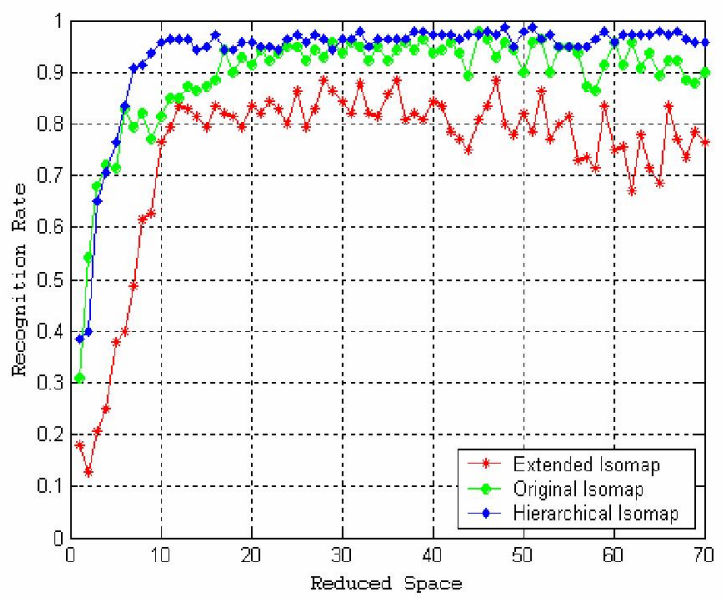

Figure 3. Recognition Rate Curve of three Isomap methods

In original Isomap, the value of nearest neighbor $k$ used is 20. In extended Isomap, the value of nearest neighbor $k w$ used in within-class matrix is 5 . In hierarchical Isomap, the value of nearest neighbor $k w$ used in within-class matrix is 5 and the value of nearest neighbor $k b$ used for calculating central point geodesic distance is 6 . The system is implemented using Matlab on a computer with a Pentium IV $(2.6 \mathrm{GHz})$ processor. 
Figure 3. indicates that the recognition rate of hierarchical Isomap is better than original Isomap [5] and extended Isomap [7]. We also compute the average recognition rate and standard deviation of recognition rate in Table 1, which shows that the proposed algorithm achieves superior performance on recognition accuracy.

Table 1. Comparison between three Isomap methods (reduced space from 10 to 70 )

\begin{tabular}{llll}
\hline Method & Parameter & $\begin{array}{l}\text { Average } \\
\text { Recognition } \\
\text { Rate }\end{array}$ & $\begin{array}{l}\text { Standard } \\
\text { Deviation of } \\
\text { Recognition } \\
\text { Rate }\end{array}$ \\
\hline $\begin{array}{l}\text { Original } \\
\text { Isomap [5] }\end{array}$ & $k=20$ & 0.9248 & 0.0320 \\
$\begin{array}{l}\text { Extended } \\
\text { Isomap [7] }\end{array}$ & $k w=5$ & 0.8044 & 0.0475 \\
$\begin{array}{l}\text { Hierarchical } \\
\begin{array}{l}\text { Isomap } \\
\text { (Proposed) }\end{array}\end{array}$ & $k b=5$, & 0.9641 & 0.0115 \\
\hline
\end{tabular}

\section{Conclusions}

In this paper, a new geodesic distance computation method is proposed for multi-class data, which computes the within-class and between-class geodesic distance with different approaches. For the within-class data, Floyd-Warshall algorithm is used to compute their geodesic distances; while for the between-class data, a novel hierarchical method is used, which hierarchically combines geodesic distance of central points of two classes and geodesic distances between within-class point and the corresponding class central point to form the geodesic distance of points belong to different classes. The final embedding result can be achieved using MDS on the hierarchical geodesic distance matrix. The experimental results show that the proposed method can achieve better embedding result for multi-class data compared with other conventional implementations of Isomap.

\section{Acknowledgements}

This work is supported by Photoelectron $+X$ interdisciplinary research funds of Dalian University of Technology, P. R. China (2006).

\section{References}

[1] I. T. Jolliffe, Principal component analysis, SpringerVerlag, New York, 1989.

[2] T. F. Cox and M.A.A. Cox, Multidimensional scaling, Chapman and Hall, London, 1994.

[3] K. V. Mardia, J. T. Kent, J. M. Bibby, Multivariate Analysis, Academic Press, London, 1979.

[4] S. T. Roweis and L. K. Saul, "Nonlinear Dimensionality Reduction by Locally Linear Embedding", Science, 2000, Vol. 290, pp. 2323-2326.

[5] J. B. Tenenbaum, V. de Silva, and John C. Langford, "A global geometric Framework for Nonlinear Dimensionality Reduction", Science, 2000, Vol. 290 , pp. 2319-2323.

[6]J. B. Tenenbaum, "Response to comments on the Isomap Algorithm and Topological Stability", Science, 2002, Vol.295.

[7] Y. M. Wu and K. L. Chan, "An Extended Isomap Algorithm for Learning Multi-Class Manifold", Proc. of ICMLC, 2004

[8] J. Wang, C. Zhang and Z. Kou, "An Analytical Mapping for LLE and Its Application in Multi-Pose Face Synthesis", Proc. of BMVC, 2003.

[9] V. N. Vapnik, The Nature of Statistical Learning Theory, Springer-Verlag, New York, 1995. 